\title{
PRECISIONES EN PASIVOS, PROVISIONES Y PASIVOS CONTINGENTES A PARTIR DE LA TEORÍA DE PROBABILIDADES
}

\author{
PRECISIONS ON LIABILITIES, ACCRUALS AND CONTINGENT LIABILITIES BASED ON \\ THE THEORY OF PROBABILITIES
}

Rosa Milagros Castañeda Moreano

Pontificia Universidad Católica del Perú

Lima, Perú

ORCID: https://orcid.org/0000-0003-3097-8504

Correo electrónico: rcastanedamoreano@gmail.com

\section{RESUMEN}

Objetivo: Describir los conceptos de pasivos, provisiones y pasivos contingentes a partir de aspectos relevantes de la Teoría de Probabilidades. Método: La investigación fue cualitativa, descriptiva, no experimental con el uso del método deductivo-inductivo, sobre la base del análisis de contenido de las Normas Internacionales de Información Financiera (NIIF) y la Teoría de Probabilidades. Resultados: Los resultados muestran discrepancias sobre los conceptos de pasivos, provisiones y pasivos contingentes, entre lo planteado por la NIC 37 y el Marco Conceptual para la Información Financiera, considerando además aspectos relevantes de la Teoría de Probabilidades. Conclusión: Existen divergencias conceptuales entre la NIC 37 y el Marco Conceptual para la Información Financiera respecto a la definición de pasivos, provisiones o pasivos contingentes, lo cual podría producir un impacto relevante en los estados financieros, así como su inadecuada interpretación.

Palabras clave: Pasivo; provisión; pasivo contingente; evento; incertidumbre.

\begin{abstract}
Objective: To describe the concepts of liabilities, accruals and contingent liabilities based on relevant aspects of the Theory of Probabilities. Method: The research was qualitative, descriptive, and non-experimental, with the use of the deductive-inductive method, based on the International Financial Reporting Standards (IFRS) content analysis and Probabilities Theory. Results: The results show discrepancies in the concepts of passives, provisions and contingent liabilities, including those raised by IAS 37 and the Conceptual Framework for Financial Reporting, also considering relevant aspects of the Theory of Probabilities. Conclusion: There are conceptual divergences between IAS 37 and the Conceptual Financial Reporting Framework in regards to the definition of liabilities, provisions or contingent liabilities, which could have a significant impact on the financial statements, as well as their poor interpretation.
\end{abstract}

Keywords: Liability; accrual; contingent liability; event; uncertainty.

( ) Los autores. Este artículo es publicado por la revista Quipukamayoc de la Facultad de Ciencias Contables, Universidad Nacional Mayor de San Marcos. Este es un artículo de acceso abierto, distribuido bajo los términos de la licencia Creative Commons Atribución 4.0 Internacional (CC BY 4.0) [https://creativecommons.org/licenses by/4.0/deed.es] que permite el uso, distribución y reproducción en cualquier medio, siempre que la obra original sea debidamente citada de su fuente original. 


\section{INTRODUCCIÓN}

En el proceso de análisis de la NIC 37 - Provisiones, Pasivos Contingentes y Activos Contingentes (en adelante NIC 37), se han identificado discrepancias en el concepto de probabilidad respecto de la Teoría de Probabilidades, con lo cual se presenta una inconsistencia en su interpretación, no solo a nivel de la NIC 37, sino también del Marco Conceptual para la Información Financiera (en adelante $\mathrm{MC}$ ) requerida para la emisión de estados financieros.

Un aspecto sujeto a análisis es aquel referido a las definiciones. Tanto la NIC 37 como el MC no establecen claramente las definiciones de pasivo, provisión y pasivo contingente, los cuales podrían ser malinterpretados. Por ejemplo, en la NIC 37, el término pasivo contingente abarca tres conjuntos diferentes de partidas que no forman una misma clase:

(i) elementos para los cuales no está claro si existe una obligación;

(ii) elementos para los cuales existe una obligación, pero presentan una alta probabilidad de flujos de recursos económicos; $\mathrm{y}$

(iii) elementos que no se pueden medir de manera fiable.

Es así que se enfocó la problemática de la adecuada definición de pasivos, provisiones y pasivos contingentes.

Se ha identificado el trabajo de Iglesias (2016), quien abordó a nivel conceptual a los pasivos de naturaleza incierta. Por otro lado, Yidan (2002) analizó las contingencias y su incertidumbre asociada para empresas públicas canadienses, con énfasis en sus prácticas de revelación, siendo estas insuficientes. De igual modo, Ribeiro (2012) analizó la práctica de gestión de resultados para empresas brasileras donde identificó el reconocimiento o no de provisiones, evidenciando la subjetividad del juicio profesional.

En relación con la incertidumbre, el MC indica las distinciones respecto a las incertidumbres alrededor de la medición, desenlace y existencia (International Accounting Standard Board, 2018). La Contabilidad de por sí no es exacta; requiere de estimaciones, las cuales no le restan utilidad a los estados financieros, siempre y cuando hayan sido efectuados con una descripción clara y precisa. Esto se encuentra en línea con el paradigma económico de la información, donde el sistema de información más útil es el que posee mayor preferencia (Cuadrado y Valmayor, 1999).

Con respecto a la existencia de una obligación, el MC indica que la "obligación debe tener el potencial de requerir que la entidad transfiera un recurso económico" (International Accounting Standard Board, 2018, párr. 4.37). Es decir, la transferencia de recursos no requiere ser cierta ni probable.

Una obligación puede cumplir la definición de un pasivo incluso si la probabilidad de transferir un recurso económico es baja. No obstante, esa baja probabilidad puede afectar decisiones sobre qué información proporcionar sobre el pasivo y cómo facilitarla, incluyendo decisiones sobre si se reconoce el pasivo ..., y cómo medirlo. (International Accounting Standard Board, 2018, párr. 4.38)

En relación con una menor probabilidad de flujo de recursos, “... la información más relevante sobre el activo o pasivo puede ser la referida a la magnitud de las posibles entradas o salidas, su calendario posible y los factores que afectan la probabilidad de que sucedan unas u otras" (International Accounting Standard Board, 2018, párr. 5.16).

De acuerdo con la NIC 37, la obligación presente proviene de un suceso ocurrido en el pasado que lo originó. A su vez, se hace mención a la consideración de toda la evidencia "disponible al final del periodo sobre el que se informa, es mayor la probabilidad de que exista una obligación presente que de lo contrario" (International Accounting Standads Board, 2020, párr. 15).

La NIC 37 hace referencia a los procesos judiciales (que involucran incertidumbre sobre su desenlace), para los cuales la determinación de la existencia de una obligación presente se efectuará al final del periodo considerando la información disponible y la opinión de expertos, reconociendo una provisión o revelando un pasivo contingente, "a menos que sea remota la posibilidad de que tenga que desprenderse de recursos que incorporen beneficios económicos..." (International Accounting Standads Board, 2020, párr. 16).

En referencia a la salida probable de recursos que incorporen beneficios económicos que permitan cancelar la obligación, la NIC 37 establece que "se considerará probable siempre que haya mayor posibilidad de que se presente que de lo contrario" (International Accounting Standads Board, 2020, párr. 23). Si no fuera probable la existencia de la obligación, será revelado en notas como pasivo contingente.

Respecto a la fiabilidad de la medición del importe de la obligación, la NIC 37 establece que, para el caso de provisiones, esta deberá ser una estimación fiable para ser reconocida en los estados financieros (International Accounting Standards Board, 2020, párr. 25). 
En referencia a la presencia de la incertidumbre y su reflejo en los riesgos, la NIC 37 indica que "para realizar la mejor estimación de la provisión, deben ser tenidos en cuenta los riesgos e incertidumbres que, inevitablemente, rodean a la mayoría de los sucesos y las circunstancias concurrentes a la valoración de la misma" (International Accounting Standads Board, 2020, párr. 42). En relación con el juicio a considerar cuando se evalúa el riesgo, asociado a condiciones de incertidumbre, la NIC 37 reconoce el efecto del riesgo por la "variabilidad en los desenlaces posibles" afectando el importe estimado de una obligación (International Accounting Standads Board, 2020, párr.43).

De acuerdo con Audi (2004), la probabilidad está definida como un valor numérico que mide el grado en el que puede esperarse que un evento ocurra. Asimismo, se encuentran en el rango 0 y 1 (García, 2015). Por ende, la Teoría de Probabilidades aporta en el análisis de la probabilidad debido a que (Castañeda, 2020):

- Es posible utilizarla para evaluar el grado de incertidumbre asociada a un fenómeno que involucra aleatoriedad, la cual se expresa entre 0 (incertidumbre) y 1 (certeza).

- Se debe revelar información suficiente en las notas a los estados financieros referente a las estimaciones y sus métodos para una estimación fiable y reducir la incertidumbre en la medición.

En ese contexto, la investigación tiene como objetivo describir los conceptos de pasivos, provisiones y pasivos contingentes a partir de aspectos relevantes de la Teoría de Probabilidades.

La investigación se justifica en la identificación de imprecisiones respecto a la incertidumbre y probabilidades, así como en la evaluación de la existencia de una obligación presente que genere una probable salida de recursos económicos, originando posibles inconsistencias en la aplicación de las NIIF.

La principal limitación fue la falta de disponibilidad de investigaciones sobre la definición conceptual de obligaciones en un entorno de incertidumbre y de los criterios de interpretación contable. Las categorías de análisis se centran en pasivos, provisiones y pasivos contingentes.

\section{MATERIALES Y MÉTODOS}

La investigación fue cualitativa, descriptiva, no experimental, deductiva-inductiva, orientada a presentar aspectos relevantes respecto de las divergencias entre los marcos normativos contables, a partir de aspectos relevantes de la Teoría de Probabilidades.

Las técnicas de recolección de datos comprendieron el análisis de contenido de la base normativa contable (NIIF) y la base teórica de la Teoría de Probabilidades.

\section{RESULTADOS}

Se ha realizado un análisis de contenido sobre la base normativa centrada en la NIC 37 y el MC en lo que respecta a los conceptos de pasivos, provisiones y pasivos contingentes; así como aspectos relevantes de la Teoría de Probabilidades.

En la Tabla 1 se presenta un resumen de las diferencias entre pasivo, provisión y pasivo contingente de acuerdo con la NIC 37, con la finalidad de presentar una visión inicial de las diferencias establecidas entre dichos conceptos como punto de partida.

Tabla 1

Diferencias entre Pasivo, Provisión y Pasivo Contingente

\begin{tabular}{lcccc}
\hline Característica & Pasivo & Provisión & \multicolumn{2}{c}{ Pasivo contingente } \\
\hline Obligación & Presente & Presente & Presente & Posible \\
Surge a raíz de sucesos pasados & $\mathrm{Si}$ & $\mathrm{Si}$ & $\mathrm{Si}$ & $\mathrm{Si}$ \\
Probable salida de recursos & $\mathrm{Si}$ & $\mathrm{Si}$ & $\mathrm{No}$ & $\mathrm{Si}$ \\
Estimación (medición) & $\mathrm{Fiable}$ & $\mathrm{Fiable}$ & $\mathrm{No}$ fiable & $\mathrm{No} \mathrm{fiable}$ \\
Hechos futuros inciertos & $\mathrm{No}$ & No precisa & $\mathrm{Si}$ & $\mathrm{Si}$ \\
Reconocida en los estados financieros & $\mathrm{Si}$ & $\mathrm{Si}$ & $\mathrm{No}$ & No \\
Vencimiento & Cierto & Incierto o cierto & Incierto & Incierto \\
Cuantía & Cierta & Incierta o cierta & Incierta & Incierta \\
\hline
\end{tabular}

Fuente: Adaptado de: "NIC 37 - Provisiones, Activos Contingentes y Pasivos Contingentes", por International Accounting Standard Board, 2020, párr. 10-30. 
La Tabla 1 muestra que, respecto de la definición de provisión, no se precisan las consideraciones vinculadas a los hechos futuros inciertos; asimismo, respecto a la definición de pasivo contingente, tampoco se precisan los criterios de la probabilidad de salida de recursos cuando se trata de una obligación posible. Se puede observar también que tanto la provisión como el pasivo contingente, poseen una cuota de incertidumbre a nivel de su vencimiento y cuantía. A continuación, se desarrolla el análisis de los conceptos de pasivo, provisión y pasivo contingente. Además, para propósitos ilustrativos, se presenta como ejemplo el caso de los procesos judiciales y su clasificación como pasivo, provisión o pasivo contingente en función al juicio aplicado en su evaluación y a sus características.

\section{Características de un Pasivo}

Según la NIC 37, las características de un Pasivo corresponden a:

- Obligación presente. La obligación es un concepto que define el compromiso, o la expectativa de cumplimiento de responsabilidad, que asume una entidad frente a terceros en un momento determinado. La obligación puede ser legal (proviene de un contrato o legislación) o implícita (se deriva de un comportamiento o política proveniente de la actividad de la industria). Es exigible y se evalúa en el momento de su registro en los estados financieros.

- Surge a raíz de sucesos pasados. La obligación presente surge de un suceso ocurrido en el pasado que determina su cumplimiento a través de la liquidación del pasivo, es decir, de la salida de recursos económicos. Son independientes puesto que no es posible evitar dichos pasivos por la gestión que pueda realizar una entidad en el futuro.

- Probable salida de recursos. Existe un alto grado de certeza o certidumbre de que la entidad incurrirá en desembolsos para efectos de liquidar la obligación.

- Estimación (medición) fiable. La estimación requiere del juicio, conocimiento y experiencia en su medición.

- Hechos futuros inciertos. No existe dependencia por parte de hechos futuros, ya sea incierto o no, debido a que su naturaleza responde a la existencia de una obligación en un momento determinado.

- Reconocido en los estados financieros. Se presenta su impacto y se revela sus características.

- Vencimiento y cuantía ciertos. La entidad tiene certeza sobre el vencimiento y el importe de la obligación.

Adicionalmente, se ha efectuado un análisis comparativo de las características de un pasivo entre lo establecido por la NIC 37 y el MC. La Tabla 2 se presenta a continuación:

En la Tabla 2, se muestran dos aspectos que podrían prestarse a una inadecuada interpretación, referidos principalmente al grado de certeza en la existencia de la obligación, así como la esencia sobre la forma:

- Grado de certeza sobre la existencia de la obligación. Referido a la incertidumbre asociada a la existencia de la obligación y a la probabilidad de salida de flujos económicos para liquidar un pasivo. Si bien el MC por un lado indica un suficiente grado de certeza,

Tabla 2

Características de un Pasivo según NIC 37 y el MC

\begin{tabular}{lcc}
\hline Características de un pasivo & NIC 37 & MC \\
\hline Obligación presente & $\mathrm{Si}$ & $\mathrm{Si}$ \\
Surge a raíz de sucesos pasados & $\mathrm{Si}$ & $\mathrm{Si}$ \\
Probable salida de recursos & $\mathrm{Si}$ & $\mathrm{Si}$ \\
Grado de certeza sobre la existencia de obligación & No precisa & Suficiente o insuficiente \\
Esencia sobre forma & No precisa & $\mathrm{Si}$ \\
Estimación (medición) & $\mathrm{Si}$ & $\mathrm{Si}$ \\
Reconocida en los estados financieros & $\mathrm{Si}$ & $\mathrm{Si}$ \\
Vencimiento & $\mathrm{Cierto}$ & Cierto \\
Cuantía & Cierta & Evaluada con fiabilidad \\
\hline
\end{tabular}

Fuente: Adaptado de: "NIC 37 - Provisiones, Activos Contingentes y Pasivos Contingentes", por International Accounting Standard Board, 2020, párr. 10-11, y de "Marco Conceptual para la Información Financiera”, por International Accounting Standard Board, 2018, párr. 4.26-4.47

12/ QUIPURAMAYOC | Vol. 29 N 59/ENERO-ABRIL, 2021 
por otro lado, si no lo hubiere, requiere la revelación de la incertidumbre asociada; sin embargo, no hace mayor precisión al respecto. Esto se vería en algún aspecto sesgado por la posición de la Gerencia frente al riesgo, lo cual influiría en las consideraciones de incertidumbre y su respectiva evaluación.

- Esencia sobre forma. Desde que la obligación puede calificarse como implícita y no solamente de carácter legal, la entidad debería considerar las prácticas del sector en el que opera y las expectativas generadas por su actividad y desempeño en la sociedad.

Como resultado del trabajo del Consejo del IASB en cuanto a la revisión de algunos conceptos del MC revisado, publicado en marzo de 2018, se hizo énfasis en la consistencia de lo siguiente:

En cuanto a la definición de pasivo, International Accounting Standard Board (2018) explica: "Una obligación presente de la entidad de transferir un recurso económico como resultado de sucesos pasados" (párr. 4.26). "Una obligación es un deber o una responsabilidad que la entidad no tiene la capacidad práctica de evitar ..." (párr. 4.29).

En cuanto al criterio revisado de reconocimiento de pasivos, se hace referencia explícita a las características cualitativas fundamentales, en cuanto a su relevancia (su reconocimiento pueda ser afectado por la baja probabilidad de un flujo de beneficios económicos o la incertidumbre en su existencia) y representación fiel (su reconocimiento pueda ser afectado por la incertidumbre en su medición, incoherencia en su reconocimiento, presentación y revelación) (párr. 5.7).

Tomando como base la NIC 37, el término "espera desprenderse de recursos" motiva a la discusión en cuanto a la interpretación que pueda tener la entidad o individuo. Para el caso de un litigio en el que ambas partes no esperan desprenderse de recursos, están sujetos a una sentencia emitida por un tribunal de justicia. Mientras tanto, no reconocerían ningún pasivo puesto que cada uno cuenta con un staff de profesionales que sustentan su posición y su entendimiento de que no esperan desprenderse de recursos. En este punto, la discusión toma un sentido más subjetivo que objetivo, y la NIC 37 debería tratar en específico este tipo de casos, pues no lo desarrolla con la precisión requerida.

Finalmente, tomando como base el MC, la obligación presente de transferir un recurso económico difiere de la obligación presente de que la entidad espera desprenderse de un recurso (esta última según NIC 37). Según Iglesias (2016):
... dado que al no ser necesario un cierto grado de certidumbre en la salida de recursos para poder hablar de pasivo, ... ya podrían reconocerse en el estado de situación financiera, aunque posiblemente con un importe casi nulo, al determinarse su valor en función de la probabilidad de salida de recursos y ser ésta muy baja. (p. 102-103)

En cuanto a la incertidumbre asociada a la obligación, el MC indica:

En algunos casos, hay incertidumbre sobre si existe una obligación. Por ejemplo, si un tercero pretende una compensación por un acto de una entidad denunciado por irregularidades, puede haber incertidumbre sobre si ocurrió o no el acto, sobre si la entidad lo cometió o sobre cómo se aplica la ley. Hasta que esa incertidumbre sobre la existencia se resuelva-por ejemplo, mediante sentencia judicial-existirá incertidumbre sobre si la entidad tiene una obligación con el tercero que pretende una compensación y, por consiguiente, si existe un pasivo ... (International Accounting Standard Board, 2018, párr. 4.35)

La incertidumbre sobre la existencia de una obligación va analizada juntamente con la probabilidad asociada a la salida de beneficios económicos, así sea ínfima que es “... necesario proporcionar en los estados financieros información explicativa sobre las incertidumbres asociadas con ello" (International Accounting Standard Board, párr. 5.14). En otras palabras, el MC sugiere efectuar una revelación de las incertidumbres asociadas con la existencia de una obligación, por más que no se cumpla con la definición de pasivo.

Si bien del análisis de la NIC 37 se concluye que un pasivo puede medirse con fiabilidad, pues los términos son ciertos, existe certeza sobre la cuantía y vencimiento, el MC considera que "para el reconocimiento de un pasivo es necesario que reúna las características fundamentales de relevancia y representación fiel” (párr. 5.7).

Con respecto a la relevancia, la incertidumbre sobre la existencia de la obligación está en línea con la probabilidad de salida de recursos, que de no existir no se reconocería el pasivo y estaría sujeta a revelación. Un proceso judicial que cuenta con una sentencia firme puede reconocerse como pasivo, puesto que existe la obligación presente y hay una certeza de salida de recursos económicos, cuyo vencimiento e importe son medibles.

\section{Características de una Provisión}

Según la NIC 37, las características de una Provisión corresponden a: 
- Obligación presente. En cuanto a que la obligación proviene de sucesos pasados y de la probabilidad de salida de recursos. Es similar a la de un pasivo.

- Hechos futuros inciertos. La NIC 37 solo hace referencia a que son reconocidos como pasivos, debido a que "representan obligaciones presentes y es probable que la entidad tenga que desprenderse de recursos, que incorporen beneficios económicos para cancelar tal obligación" (International Accounting Standards Board, 2020, párr. 13(a)). Por otro lado, la NIC 37 (2020) indica que:

No existe dependencia en la existencia de la obligación por parte de hechos futuros, ya sea incierto o no, debido a que su naturaleza responde a la existencia de una obligación en un momento determinado y no a su posible situación en el futuro. (párr. 18-19)

- Reconocimiento en los estados financieros. Presentación del impacto y revelación de sus características, puesto que implica una probable salida de recursos económicos y puede ser medido con fiabilidad.

- Vencimiento y cuantía inciertos. La entidad no tiene certeza sobre el vencimiento de la obligación y su importe. De este modo, hay una inconsistencia entre los requerimientos como pasivo, en cuanto a la fiabilidad de su medición y la incertidumbre de su cuantía según el párrafo 7 de la NIC 37.

Sin embargo, la principal diferencia en relación con una provisión es que esta presenta vencimiento y cuantía no exactos, pero si estimables. Por ejemplo, en el caso de la provisión por cierre de mina, una empresa por lo general efectúa proyecciones de los recursos (materiales, flujos, entre otros) que se espera desprender a efectos de dejar la zona de extracción de mineral en las mismas condiciones en las que se encontró. Para ello se realiza la estimación, considerando su mejor criterio, experiencia y el apoyo de expertos, de cuánto sería la vida útil de la mina, la tasa de descuento para estimar la provisión y su respectiva actualización cada año.

Según lo dispuesto por la NIC 37, se puede identificar que una provisión presenta características similares al de reconocimiento como pasivo, siempre que cumpla los requerimientos como tal (obligación presente, originada por sucesos pasados y probable salida de recursos) y sea medible con fiabilidad, aunque su vencimiento y cuantía no sean exactos. En ese sentido, la NIC 37 debería marcar distancia cuando menciona que su reconocimiento es similar al de un pasivo, puesto que la incertidumbre en la existencia de la obligación afecta a su condición de obligación presente, más aún cuando existe discrepancia de este punto en el MC. En el caso del proceso judicial, aún sin tener una sentencia, se reconocerá una provisión siempre que exista la obligación y su importe pueda ser estimable.

\section{Características de un Pasivo contingente}

Si se trata de una obligación presente con una probable salida de recursos pero que no pueden ser medidos con fiabilidad, solo se trataría de un pasivo contingente según la NIC 37. Por otro lado, se establece además que el pasivo contingente es una obligación posible y surge cuando la existencia de una obligación presente es incierta, pero algún suceso futuro confirmará si la entidad tiene esa obligación (sección Definiciones, párr. 10). A fin de establecer precisiones, la NIC 37 debería centrarse en la noción de la incertidumbre que rodea al análisis en la determinación de si existe o no una obligación, puesto que su estimación puede llevarse a cabo con diferentes instrumentos, como por ejemplo: valor esperado, flujo de caja esperado, entre otros. Siguiendo el ejemplo de un proceso judicial, donde aún no hay sentencia y se tiene conocimiento de una incertidumbre asociada tanto a la existencia de la obligación como a su medición, siempre que sea remota, será revelada en notas como pasivo contingente.

A nivel de la Teoría de Probabilidades hay una diferencia entre suceso y evento. Según Toma y Rubio (2012), suceso "es el conjunto de todos los resultados posibles de un experimento aleatorio" (p. 209). Por otro lado, evento "es un subconjunto de un espacio muestral" y se entiende como el que agrupa resultados con una misma característica (p. 210). No obstante, autores como Spiegel, Schiller y Srinivasan (2013) sostienen que suceso y evento pueden ser entendidos en el mismo sentido (p. 4).

$\mathrm{Al}$ respecto, es importante tener una interpretación clara de lo que se entiende por suceso o evento a nivel de la NIC 37. El término suceso como traducción del término en inglés event, se interpreta como un hecho o acontecimiento a lo largo de la NIC 37. El párrafo 23 de la NIC 37 hace referencia a la probabilidad de la ocurrencia de un evento, siendo coherente con la Teoría de Probabilidades. Por otro lado, la Teoría de Probabilidades considera aspectos como riesgo e incertidumbre, siendo que el aporte de esta teoría es su rigurosidad matemática y su interpretación, lo cual permite afinar los conceptos que aborda la NIC 37, respecto de lo siguiente:

(i) El concepto de incertidumbre tiene un efecto importante en la determinación de la existencia de una obligación, puesto que es un aspecto relevante para la definición de un pasivo. La incertidumbre es la carencia de certeza sobre algo, lo cual está asociado con experimentos aleatorios, cuyos resultados no pueden predecirse con seguridad. 
(ii) El entendimiento de suceso o evento. Para efectos del reconocimiento de probabilidad, es necesario calcular la probabilidad del evento para su estimación, como por ejemplo la probabilidad de salida de recursos económicos.

(iii) El concepto de probabilidad, como la estimación de un número que representa un amplio rango de posibilidades, expresado entre 0 y 1 , de la ocurrencia de un evento, más que de considerar la probabilidad como "más probable que ocurra a que no ocurra" (International Accounting Standard Board, 2020, párr. 23). La probabilidad adquiere relevancia pues es considerado al momento de evaluar la probabilidad que exista una obligación presente, así como la probabilidad de que la entidad se desprenda de recursos económicos.

Como resultado del análisis efectuado, la Tabla 3 propone las siguientes precisiones a ser incorporadas en la NIC 37:

En ese sentido, se entiende que existe incertidumbre sobre el desenlace (importe o cronograma de flujo de recursos económicos, es decir baja probabilidad del evento salida de recursos) o incertidumbre sobre su medición (cuando los importes monetarios se estiman).

Finalmente, a nivel de definición de los términos pasivos, provisiones y pasivos contingentes, no se han identificado criterios claros y precisos por lo que se presenta una divergencia entre lo planteado por la NIC 37 y el MC, las cuales podrían generar errores en la interpretación, en el tratamiento contable e inclusive podrían producir la ausencia de registro contable.

\section{DISCUSIÓN}

Los resultados de la investigación a nivel descriptivo muestran diferencias en ciertas precisiones sobre los conceptos de pasivos, provisiones y pasivos contingentes, entre lo planteado por la NIC 37 y el MC, considerando aspectos relevantes de la Teoría de Probabilidades.

En efecto, en relación con el concepto de pasivo, los resultados indican que la obligación presente de transferir un recurso económico tiene un carácter de expectativa en la NIC 37, e inevitable en el MC. A la luz de la Teoría de Probabilidades, un evento representa las características similares de los resultados obtenidos de acuerdo a lo señalado por Millones et al. (2018), Toma y Rubio (2012) y Spiegel et al. (2013). En ese sentido, el entendimiento de suceso o evento en la NIC 37 hace referencia a un hecho o acontecimiento, mientras que la probabilidad de la ocurrencia de un evento expuesto por el párrafo 23 coincide con la Teoría de Probabilidades, aspecto necesario para evaluar la probabilidad de salida de recursos económicos. Otro aspecto relevante es el nivel de certidumbre sobre la existencia de una obligación, característica que define a todo pasivo. El MC señala que la existencia de la obligación está vinculada a la probable salida de recursos económicos, refiriendo a que puede tener certeza suficiente, y de no tenerla, cabe la revelación de la incertidumbre asociada sin impactar los estados financieros. En cambio, la NIC 37 solo indica que la existencia de una obligación se efectúa con información disponible, que incluye la opinión de expertos, con lo cual se evidencia divergencia en ese sentido.

Tabla 3

Precisiones para incorporar a los conceptos de Pasivos, Provisiones y Pasivos contingentes

\begin{tabular}{|c|c|c|}
\hline Pasivo & Provisiones & Pasivos contingentes \\
\hline $\begin{array}{l}\text { - Obligación presente de transferir un re- } \\
\text { curso económico. } \\
\text { - Dicha obligación debe cumplir con las } \\
\text { condiciones de la realidad económica } \\
\text { más que su forma legal, siendo legal o } \\
\text { implícita. } \\
\text { - La obligación presente surge a raíz de } \\
\text { eventos pasados, que determinan su li- } \\
\text { quidación, independientemente de accio- } \\
\text { nes futuras. } \\
\text { - Requiere una salida de recursos econó- } \\
\text { micos, lo cual se traduce en un nivel de } \\
\text { certeza. } \\
\text { - Su medición es fiable en cuanto al impor- } \\
\text { te y vencimiento de la obligación. } \\
\text { - La existencia de la obligación requiere de } \\
\text { certeza. }\end{array}$ & $\begin{array}{l}\text { - Obligación presente de transferir un re- } \\
\text { curso económico. } \\
\text { - Dicha obligación debe cumplir con las } \\
\text { condiciones de la realidad económica } \\
\text { más que su forma legal, siendo legal o } \\
\text { implícita. } \\
\text { - La obligación presente surge a raíz de } \\
\text { eventos pasados, que determinan su li- } \\
\text { quidación, independientemente de accio- } \\
\text { nes futuras. } \\
\text { - La existencia de la obligación requiere de } \\
\text { un alto nivel de probabilidad. } \\
\text { - Presenta vencimiento y cuantía no exac- } \\
\text { tos, pero si pueden ser estimables con } \\
\text { fiabilidad. }\end{array}$ & $\begin{array}{l}\text { - Si se trata de una obligación presente de } \\
\text { transferir un recurso económico, se pue- } \\
\text { de presentar dos situaciones: } \\
\text { (i) no es probable que la entidad se des- } \\
\text { prenda de recursos, o } \\
\text { (ii) no es fiable su medición. } \\
\text { - Si se trata de una obligación posible, que } \\
\text { está sujeta a sucesos futuros inciertos, } \\
\text { debido a la incertidumbre en su existen- } \\
\text { cia. Es decir, hay una alta probabilidad de } \\
\text { que no exista la obligación e implica el } \\
\text { cálculo de la probabilidad de eventos. }\end{array}$ \\
\hline
\end{tabular}

Fuente: Elaboración propia 
Respecto al concepto de provisiones, se identifica la misma observación que para el pasivo en relación con la obligación presente de transferir un recurso económico. Por otro lado, se identifica que en el caso de provisiones existe un alto grado de probabilidad sobre la existencia de una obligación, referido a la incertidumbre asociada a la existencia de la obligación reflejada en la probabilidad de la salida de recursos económicos para liquidar dicha obligación, lo cual no presenta mayor detalle en la NIC 37; quedando sujeto a la mejor interpretación de la entidad en cuanto a su análisis.

Respecto al concepto de pasivos contingentes, aquellos que provienen de obligaciones presentes, la incertidumbre sobre el desenlace conlleva a la baja probabilidad de la salida de recursos; mientras que aquellos pasivos contingentes provenientes de obligaciones posibles están sujetos a la incertidumbre de la existencia de dicha obligación, lo cual está reflejado en una alta probabilidad de que no exista la obligación. No queda claro cuál es el límite de probabilidad que determina si una obligación es calificada como presente o posible. Con respecto a ello, los trabajos de Iglesias (2016), Yidan (2002) y Ribeiro (2012) permitieron identificar aspectos de análisis conceptual y de suficiencia en la revelación, lo cual refuerza el hecho que la presente investigación contribuye con un análisis de mayor profundidad sobre dichos conceptos.

En conclusión, se han identificado divergencias que podrían generar errores en la interpretación, con respecto al tratamiento contable de pasivos, provisiones o pasivos contingentes, e inclusive podría producir la ausencia de registro contable; lo cual generaría un impacto relevante en los estados financieros, así como una inadecuada interpretación de sus estados financieros. Por lo anteriormente expuesto, el organismo emisor de las normas IASB debe efectuar una revisión de la NIC 37.

\section{REFERENCIAS}

Audi, R. (Ed.). (2004). Diccionario Akal de Filosofía. (H. Marraud, y E. Alonso, Trads.) Madrid: Ediciones Akal, S.A.

Castañeda, R. (2020). Criterios de la teoría de probabilidades en la evaluación de contingencias en empresas mineras peruanas. Quipukamayoc, 28(58), 51-58. https:// doi.org/10.15381/quipu.v28i58.19262

Cuadrado, A., y Valmayor, L. (1999). Teoría Contable. Metodología de la Investigación Contable. Madrid: McGraw-Hill Interamericana de España.
García, M. A. (2015). Introducción a la Teoría de la Probabilidad (Vol. Primer curso). México, D.F.: Fondo de Cultura Económica.

Iglesias, S. (2016). Reforma de la NIC 37: un contraste conceptual de los nuevos desarrollos normativos sobre provisiones (Tesis doctoral). Universidad Complutense de Madrid, Madrid. Recuperado de: https://eprints.ucm. es/40624/1/T38186.pdf

International Accounting Standards Board. (2015). Normas Internacionales de Información Financiera. Parte A. Londres: IFRS Foundation.

International Accounting Standard Board. (2018). Marco Conceptual para la Información Financiera. Consejo Normativo de Contabilidad. Recuperado de: https:// www.mef.gob.pe/contenidos/conta_publ/con_nor_ co/AnnotatedRB2019_A_ES_cf.pdf

International Accounting Standard Board. (2020). Consejo Normativo de Contabilidad - NIIF. Consejo Normativo de Contabilidad. Recuperado de: https://www. mef.gob.pe/contenidos/conta_publ/con_nor_co/nic/ SpanishRed2020_IAS37_GVT.pdf

Millones, R., Barreno, E., Vásquez, F., y Castillo, C. (2018). Estadística Descriptiva y probabilidades. Aplicaciones en la ingeniería y los negocios. Lima: Universidad de Lima. Fondo Editorial.

Ribeiro, A. (2012). Provisões, Contingências e Normas Contábeis: Um Estudo de Gerenciamento de Resultados com Contencioso Legal no Brasil (Tesis de maestria). Universidade De São Paulo, São Paulo. Recuperado de: https://www.teses.usp.br/teses/disponiveis/96/96133/ tde-17052012-114448/publico/AntonioCRibeiro_Corrigida.pdf

Spiegel, M., Schiller, J., Srinivasan, R. (2013). Probabilidad y Estadística (4ta ed.) México D.F.: McGraw-Hill/Interamericana Editores, S.A. DE C.V.

Toma, J., y Rubio, J. (2012). Estadística Aplicada. Primera Parte (2a. ed.). Lima: Universidad del Pacífico, Centro de Investigación.

Yidan, B. (2002). Litigation Contingency Disclosures by Canadian Public Companies (Tesis doctoral). Saskatoon. Recuperado de: https://harvest.usask.ca/bitstream/ handle/10388/etd-07232009-105147/Chui_Yidan_ sec_nc_2002.pdf?sequence $=1$ \&isAllowed $=y$ 